\title{
Design and Develop an Autonomous UAV Airship for Indoor Surveillance and Monitoring Applications
}

\author{
Hairol Nizam Mohd Shah", Mohd Zamzuri Ab Rashid", Zalina Kamis", Mohd Shahrieel Mohd Aras", \\ Nursabillilah Mohd Ali\#, Faizil Wasbari", Mohd Nor Fakurazy Bin Abu Bakar" \\ \# Center for Robotics and Industrial Automation, Faculty of Electrical Engineering, Universiti Teknikal Malaysia Melaka, Malaysia \\ *Faculty of Mechanical Engineering, Universiti Teknikal Malaysia Melaka, Malaysia \\ E-mail: $\underline{\text { hnizam@utem.edu.my }}$
}

\begin{abstract}
This project is about to develop an airship based on small size remotely controlled by human. The airship is one of Unmanned Airship Vehicle (UAV) which is can be apply in advertising, VIP security inspection, traffic monitoring and management and so on. The main purpose of this project is to design and develop an autonomous UAV airship for indoor surveillance and monitoring applications. The image will be captured from wireless camera where it mounted at a bottom of gondola. To determine the centroid points of the object are implemented in three phase edge detector, canny operator and threshold. The object will be display on Graphical User Interface (GUI) in 2D coordinated. In this project the systems able to detect only one object at one time.
\end{abstract}

Keywords - canny operator, graphical user interface (GUI), object detection, edge detector

\section{INTRODUCTION}

Today, the airship is capable to supporting terrestrial border protection as well as maritime and surveillance. The airships are designed to allow for operation flexibility. The mission need and operation concept will greatly determine the particular configurations of the individual airship and the overall fleet delivered. In order to effectively support the generals need to national border security system, our airship are designed to handle a made range of climatic conditions and payload demands. Unmanned Airship Vehicle (UAV) is an aircraft without human pilot onboard. It designed that can be controlled from a remote location by a human pilot on the ground, or it can fly autonomously based on the preprogrammed flight plans using the automations system.

One of the basic characteristic of an UAV is that it must be equipped with the payload to carry out its target missions. Hence, if the aircraft equipped with a digital camera for taking aerial photos, then it can be considered an UAV. The UAV are often utilized for high risked mission or mundane monitoring. Currently, most of the UAV's are used for military purposed such as to performed reconnaissance as well as attack missions. In addition to that, they are also used in a small but growing numbers of civil applications such as fire fighting, crop dusting and aerial photography.

In this project we propose to design and develop an autonomous UAV airship for indoor surveillance and monitoring applications. The airship was equipped with wireless camera and wireless transmission to allow basic navigation down the hallways. The monitoring and detection will be based on multi-input received from the intelligent and active vision implemented in the UAV airship. The feedback and monitoring output data from the control panel will be transmitted via wireless communication to the UAV.

\section{LITERATURE REVIEW}

The previous research, [1,2] the project goal that they developed are provide a low speed aerial vehicle for environmental monitoring missions. Among the project objectives, one is to develop a realistic visual simulator of the airship from take off flight and landing. Flight simulator is systems that tries to copy, or simulate the experience of flying an aircraft. It is meant to be as realistic as possible. The different types of flight simulator range from computer based games up to full-size cockpit replicas mounted on hydraulic (or electromechanical) actuators, controlled by state of the art computer technology [3].

They also make a priority to dynamic modeling and control system. Another previous make a research of the airship mostly focused on manual controller and they are also make a complete airship dynamic model based on body coordinated framed [4] .The equation that they make to make a stability of dynamic model is given by : 
The added mass and inertia terms $\left(\lambda_{j}\right)$ are included in $M_{\text {add }}$ show in Eq. 1.

$$
M_{\text {add }}=\left|\lambda_{j}\right| \quad i, j=1,2 \ldots . n
$$

The kinetic energy (T) of inertia can be written as:

$$
T=\frac{1}{2} \sum_{i=1}^{6} \sum_{j=1}^{6} \lambda_{j} \zeta_{i} \zeta_{j}
$$

The momentum (D) of the inertia can be written as:

$$
D_{i}=\frac{\partial T}{\partial \zeta_{i}} \quad i=1,2 \ldots n
$$

The airship dynamic model is based on the body coordinated frame which is not inertia coordinated frame. So the inertia force and momentum should be interconvert for coordinated gravity (CG) to coordinated vision (CV) [5] are show in Eq. 4 and 5.

$$
\begin{aligned}
F_{1}= & {\left[F_{x}+F_{y}+F_{z}\right]^{T}=-\left(\frac{a E}{d t}+\Omega x E\right) } \\
M_{1} & =\left[M_{x}+M_{y}+M_{z}\right]^{t} \\
& =-\left(\frac{a K}{d T}+\Omega x K+V x E\right)
\end{aligned}
$$

The other research [2] also make a assumption for the dynamic modeling and control system to the airship that have been developed. The type of the airship dynamic modeling is given by this Eq. 6:

$$
M_{v}=F_{d}(v)+A(v)+G+P
$$

Where:

$$
\begin{aligned}
& M=\text { Inertia matrix } \\
& v=\text { vector of linear and angular velocity } \\
& F_{d}=\text { vector of centrifugal forces } \\
& A=\text { vector of aerodynamical forces } \\
& G=\text { vector of gravitional forces }
\end{aligned}
$$

They are many type that the graphical user interface that used. Graphical user interface is a type of user interface item that allows people to interact with programs in more ways than typing such as computers. For the examples, they used External Authoring Interface (EAI) [5].EAI is an interface that allow program written in Java and JavaScript to control the contents of a VRML world. The control available includes the object creation and removing, operation as rotation, translation, user point of view changing and also properties changing. This interface is difficult to apply because we need to understand the VRML world.

There are also used the human-machine interface (HMI) to provides the communication and visualization mechanism between the operator and the navigation system on the board of airship. The other method is used the external web interface (EWI) for the project [6]. Figure 1 shows the example of possibilities of the web interface that they used. After logging on, the authorized user will be able to watch picture transmitted from web camera and to control the airship by means of several buttons or keyboard arrows. Moreover, it will be informed about the state of the battery.

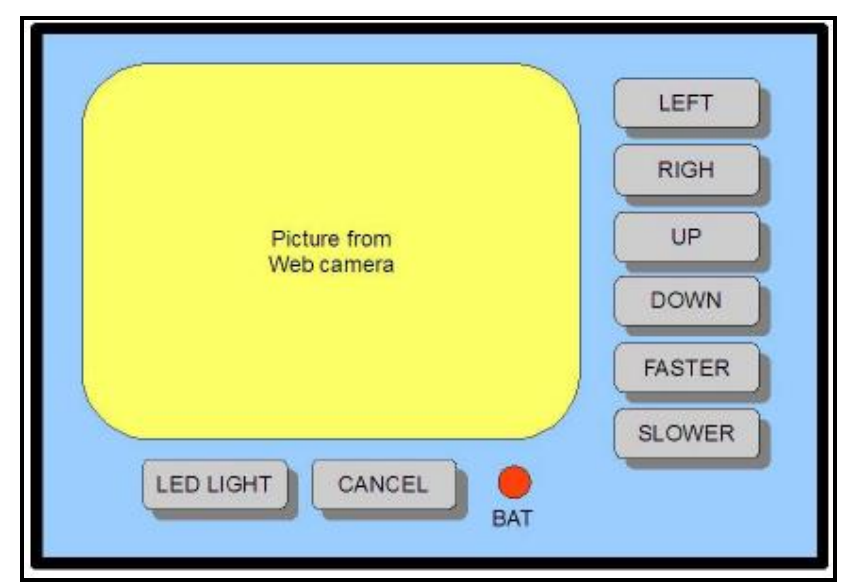

Fig. 1 Possibilities of the web interface

In the years since the war, airships have seen a decline in popularity and usage. In present day, airships are typically used for advertising, sightseeing, surveillance, and research. Figure 2 shows a timeline of airship development starting in the 1850s with Henri Giffard's first engine powered dirigible and ending in 1960s.

The purpose of the project is to develop the system that able to recognized object that it detects by the camera. This project is dividing into two parts that is hardware and software. In the hardware part, a camera will be installing in a airship that it can capture the image from the driver view. The video information about the object that has been capture will be transmitted from camera to PC and it precedes the image analysis and recognition by the specific software.

In the software part, MATLAB is used such as Image Acquisition Toolbox and Image Processing Toolbox. This toolbox is used as a library to select the suitable algorithms inside the toolbox which can be applied to recognize the object from the input images.

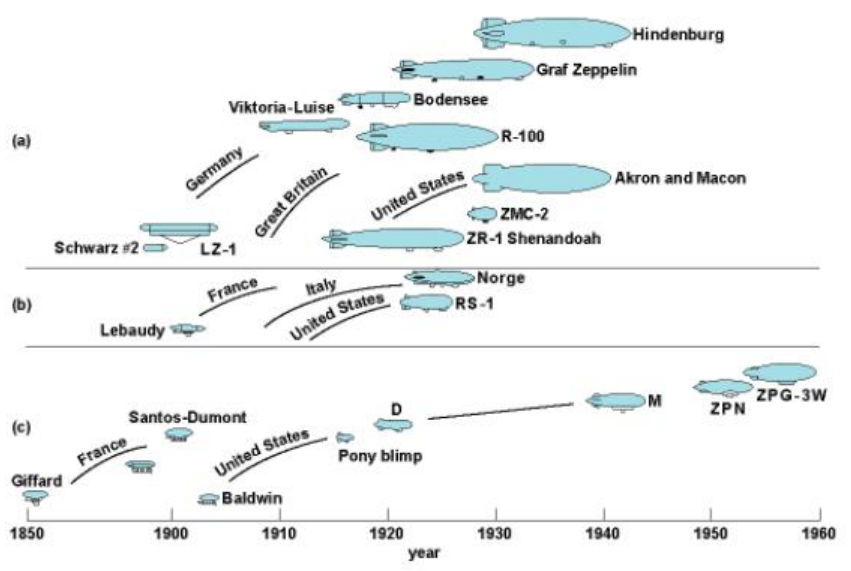

Fig. 2 History of Airship Development (a) Rigid Airships (b) Semi-rigid Airships (c) Non-rigid Airships 


\section{METHODOLOGY}

Figure 3 shows the project implementation flow chart. The general step in project methodology, literature study is the first step that must be used and done to collect the data about the monitoring system. Then the programming software is identifying to developed algorithm and design the Graphical User Interface (GUI) to solve the monitoring system.

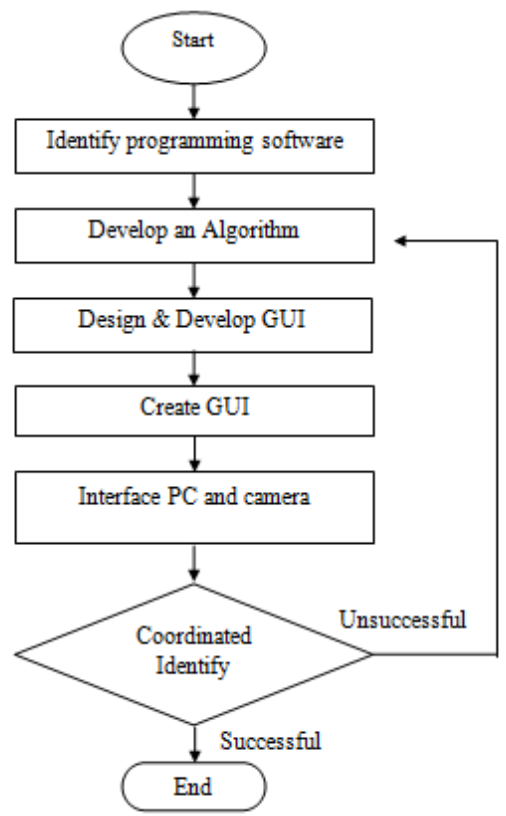

Fig. 3 Flow of the Project Methodology

\section{A. Develop detection and tracking algorithm}

The first step in the detection and tracking algorithm is to recognize image objects captured by the camera. Then, determine the object from the image that has been captured by wireless camera. After detect the object, the centroid of this object is determined. This centroid is then compared with the center of the image to get the coordinate at $\mathrm{X}$-axis and y-axis. Figure 4 shows the flowchart for the detection and tracking algorithm.

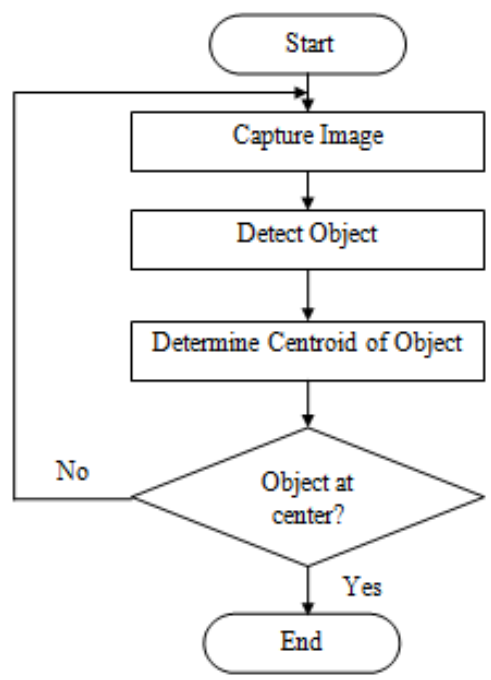

Fig. 4 Detection and tracking algorithm flowchart

\section{B. Image Processing}

\section{Edge detector}

Edge detection that are commonly used to trace a certain object that have a partial part and the detector that use have a several function to its requirement [7-12]. The basic edgedetection operator is a matrix area gradient operation that determines the level of variance between different pixels. The edge-detection operator is calculated by forming a matrix centered on a pixel is classified as an edge.

\section{Canny Operator}

Canny edge detection algorithm is known to many as the optimal edge detector [13-14]. The Canny edge detector is a first smoothes the image to eliminate and noise. The operator finds the image gradient to highlight regions with high spatial derivatives. Then it detect the regions and suppresses any pixel that is not at the maximum (non maximum suppression). The gradient array is now further reduced by hysteresis.

Hysteresis is used to track along the remaining pixels that have been suppressed. There are two threshold can be applied with three threshold condition; (1) the magnitude is below that the first threshold, it is set to zero (made a non edge), (2) the magnitude is above the threshold, it is made an edge and (3) the magnitude is between the two thresholds, then it set to zero unless there is a path from this pixel to a pixel with a gradient above T2.

Canny detector works in a multi-stage process. The first stage will start with the image itself, the image is smoothed by Gaussian convolution. Then a simple 2-D first derivatives operator is applied to the smoothed image to highlight regions of the image with high spatial derivatives. Edges give rise to ridges in the gradient magnitude image [15-16].

\section{Thresholding}

A simple and reliable method for image segmentation, thresholding is used to convert grayscale images to binary images by setting pixels with intensities values below or above the threshold value to black or white respectively [1721]. Figure 5 shows a visual example of thresholding.

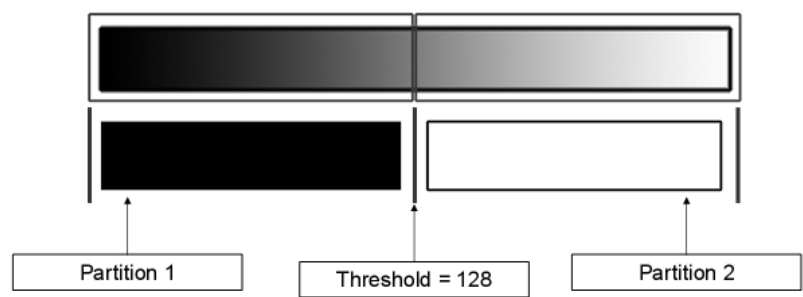

Fig. 5 Example of thresholding

The threshold value can be determined by analyzing the image's histogram. A histogram is a graphical representation of the intensity distribution in a digital image. The normal way of obtaining the threshold value is to use the peak and valley method, where the two highest peaks are found and taking the intensity value of the deepest valley between the two peeks. Figure 6 shows an example of using this method on an image to get a segmented image. 


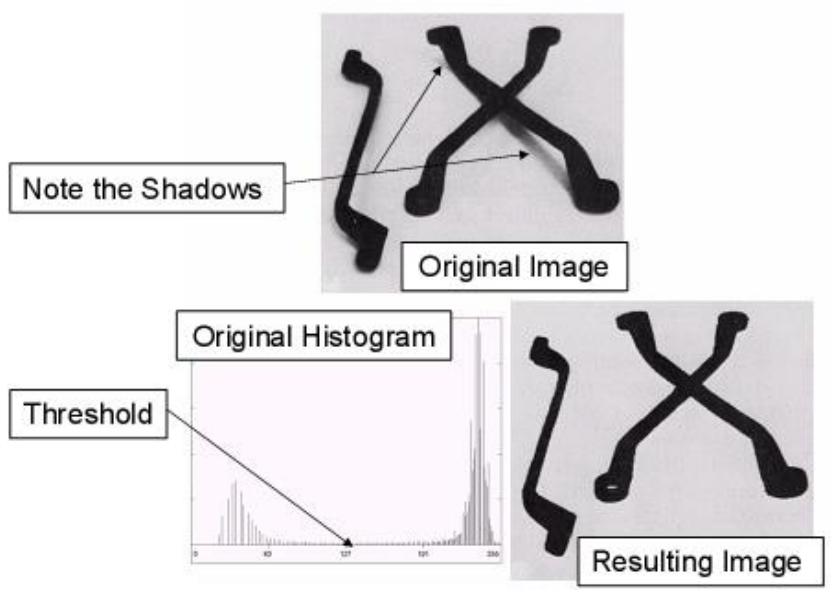

Fig. 6 Example of thresholding

\section{Integrated with $\mathrm{PC}$ ports}

MATLAB provides support to access parallel port (also called as printer port or LPT port) and serial port (also called COM port) of a PC [22-24]. For this project, it used parallel port to connected PC and hardware. The parallel port is the most commonly used port for interfacing homemade projects. This port will allow the input of up to 9 bits or the output of 12 bits at any one given time, thus requiring minimal external circuitry to implement many simpler tasks. The port is composed of 4 control lines, 5 status lines and 8 data lines. It's found commonly on the back of the PC as a D-Type 25 Pin female connector. Figure 7 shows the basic diagram of the parallel port.

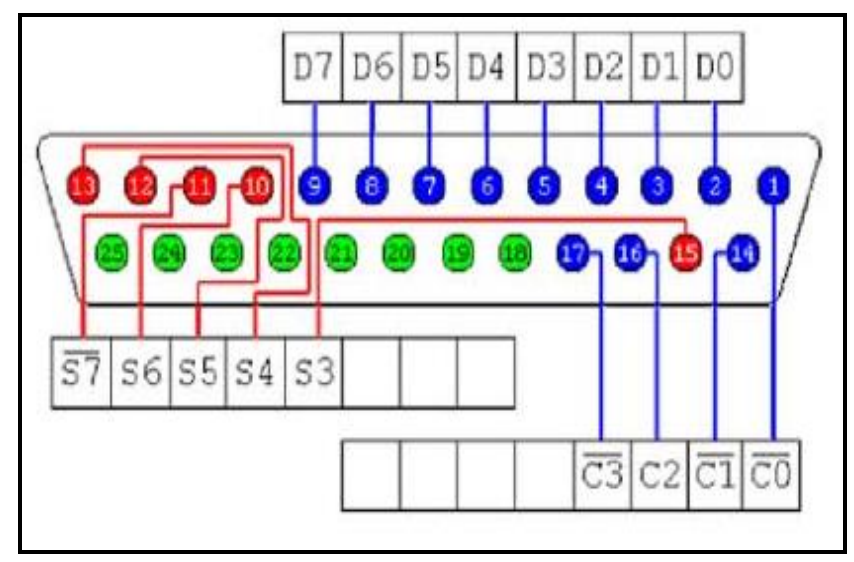

Fig. 7 Basic diagram of the parallel port

D. Main Components of the Airship

The main components of the airship can be divided into 6 components which are gasbag (Envelope), gondola (Console casing), propulsion system, surveillance system, power source and empennage (Tail fins stabilizers).

\section{Structural category and shape of the gas envelope}

\section{Structural Category}

Considering all factors, the non-rigid structure is chosen for the gas envelope. With this non-rigid structure, it is known as the pressure airship. The pressure of the lifting gas within maintains the hull profile.

\section{Shape of gas envelope}

The shape of the envelope has a major influence on its overall performance. Envelope weight is proportional to surface area and lift is proportional to the volume. In an ideal case, the surface area should be as small as possible relative to the volume. Air resistance is also determined primarily by the surface area. A spherical shape provides optimum lift efficiency. Any deviation from this optimum should utilized shapes based on circular arc sections to minimize surface stress.

\section{Shape of Gondola}

The shape must be of a streamline body as such drag is reduced. It must not have sharp corners or rough surface. This is to reduce both flow separation (results in wake or turbulence formed, increasing the drag force) and skin friction drag. It must be able to house all the components. To meet the criteria, Figure 8 shows the shape of gondola where model A will be selected as the gondola for the airship.

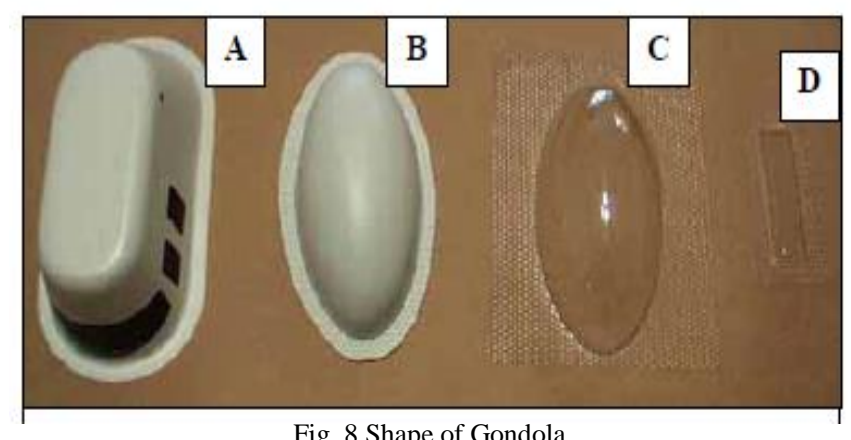

Fig. 8 Shape of Gondola

\section{Propulsion System and Surveillance System}

\section{Propulsion System}

To fulfil the operation criteria, this design will require a minimum of three motors fixed with propellers. To allow reverse thrusting, 6-9 propellers are fixed to the rotating shaft of the motors. The line of thrust must be variable to produce the pitching motion.

\section{Surveillance System}

Wireless video camera with a built-in transmitter is to be installed into the system. The direct line of sight must be variable. It is to have a visual capability of $300 \mathrm{~m}$ unobstructed.

\section{Empennage (Tail fins stabilizers)}

The bare hull of streamline form is directionally unstable, tending always to turn broadside on to the direction of motion. Tail fins are required to ensure the stability of the airship. They also act as flow straighteners, arranged in a cross configuration. Control surfaces (elevators, rudders) will not be installed on the tail fins, as they are not required to generate a pitching moment. Moving at a slow speed will also render the control surfaces ineffective. Figure 9 shows the position of gondola and motors. 


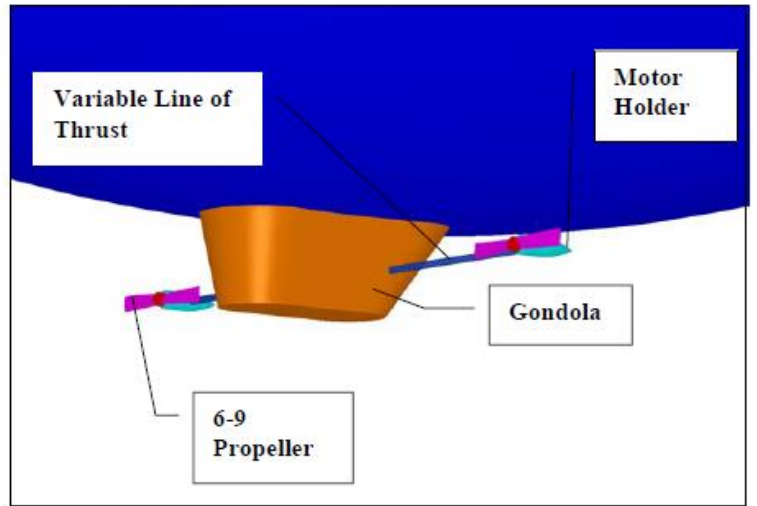

Fig. 9 Gondola and motors position

\section{RESULT}

To quantify the prototype as a UAV surveillance airship, the resolution of the image transmitted back to ground operator need to be determined. With the resolution known, the user can foresee the smallest object that can be detected at a specific range. If the dimension of the object to be survey is known, the flight altitude of the airship can be controlled such that the object remains detectable at the operating flight altitude. The experimental setup for tabulating resolution is show in Figure 10.

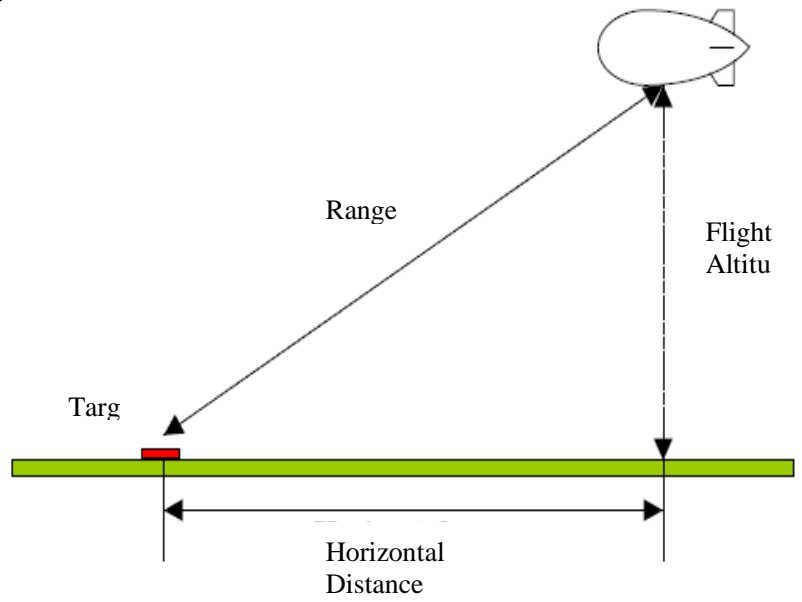

Fig. 10 Experimental setup for tabulating resolution

\section{A. Object Detection}

To test the detection algorithm, two different objects with different threshold are used. The images contain different object likes triangular and other shape by using 1.0 threshold. The result for triangular and other object is shows in Figure 11 and 12.

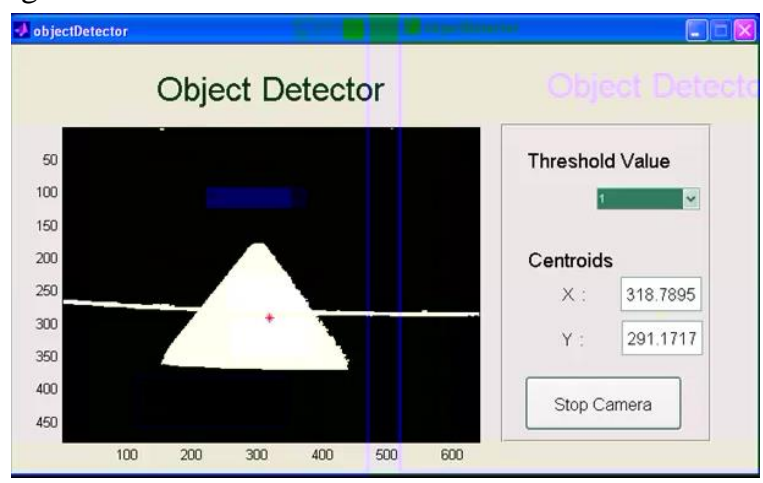

Fig. 11 Test for triangular object

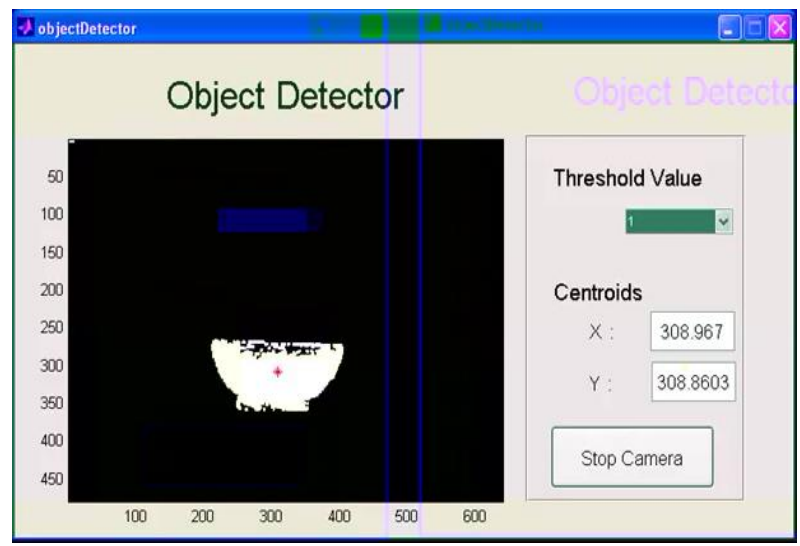

Fig. 12 Test for other object

B. Thresholding for segmentation

There are same images shown with two threshold value for this object. Figure 13 and 14 shows the different image but same object using two value of threshold. To get the best results, the threshold value was set manually.

C. Developed Graphical User Interface (GUI)

After segmenting out the object, the centroid of the object is determined. This centroid is used to determined whether the object is located based on the appeared $\mathrm{x}$-axis and $y$-axis [25-27]. Next, a graphical user interface was developed to support the live feed from the camera for real time processing and tracking. Figure 15 shows the GUI that developed.

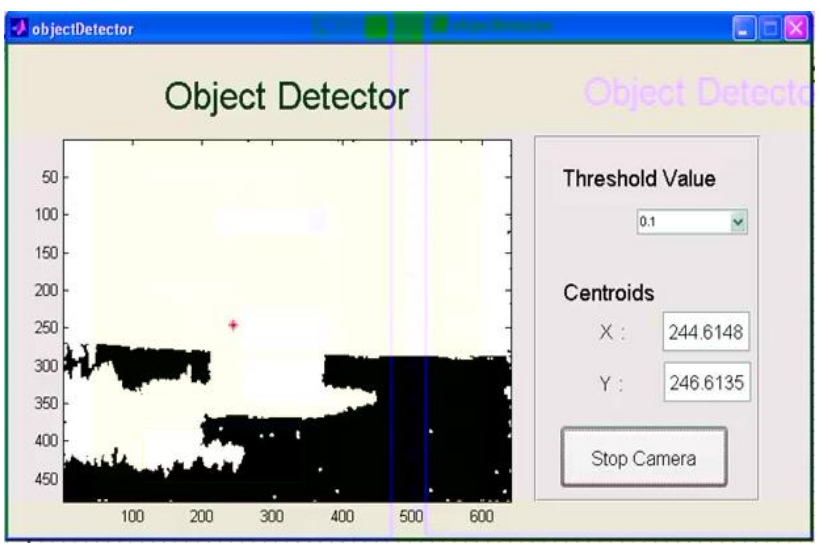

Fig. 13 Segmentation of image using threshold 0.1

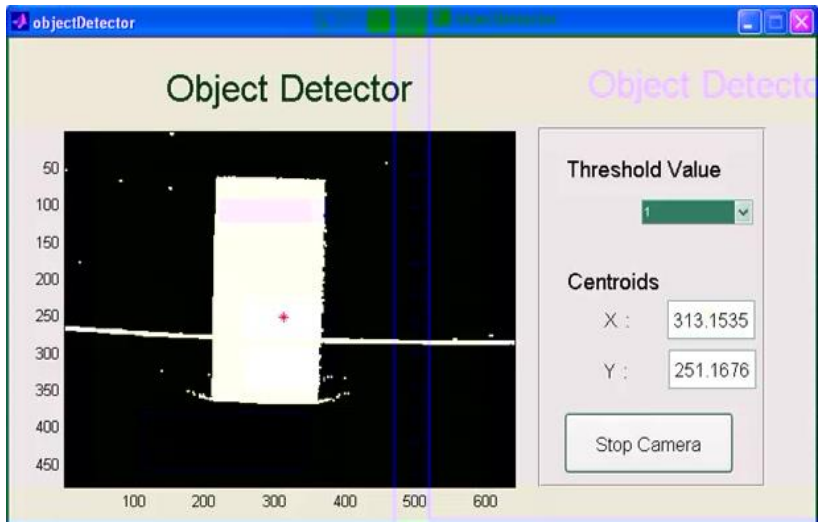

Fig.14 Segmentation of image using threshold 1 


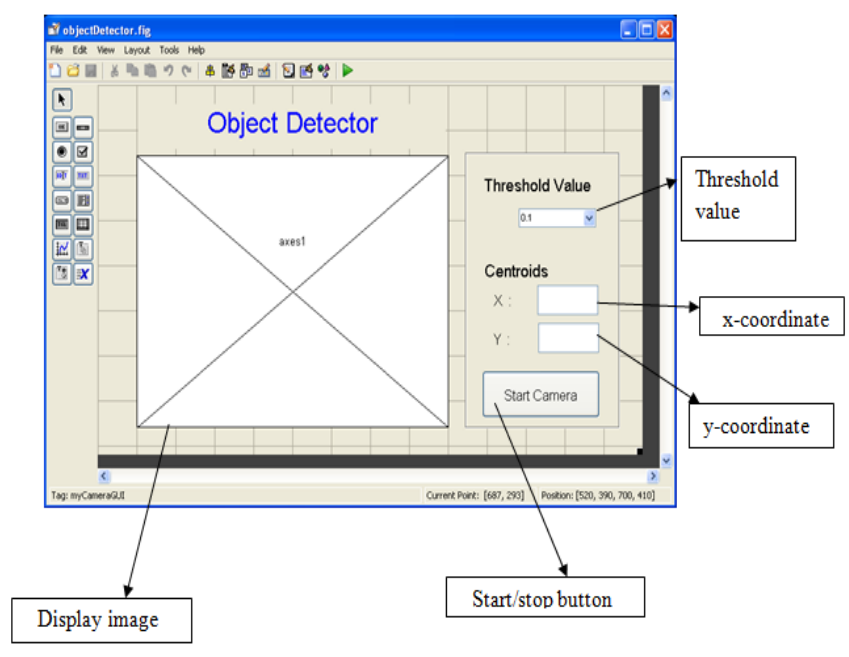

Fig. 15 GUI for real time processing and tracking

\section{DISCUSSION}

From the results obtain a segmentation consisting of Canny edge detection. The advantages of Canny edge detector is it using the probability for finding error rate, improving signal to noise ratio. The canny operators also make better detection especially in noise condition. Thresholding is the simplest method of image segmentation. From a grayscale image, thresholding can be used to create binary images. During the thresholding process, individual pixels in an image are marked as "object" pixels if their value is greater than some threshold value (assuming an object to be brighter than the background) and as "background" pixels otherwise. This convention is known as threshold above. Typically, an object pixel is given a value of " 1 " while a background pixel is given a value of " 0 ." Finally, a binary image is created in each pixel white or black, depending on a pixel's label.

\section{CONCLUSIONS}

As conclusion, the main goal of this project is to design and developed an autonomous UAV airship for indoor surveillance and monitoring applications. The system can recognized the object and display the object centroid in 2D coordinated at Graphical User Interface (GUI). The result show the system able to detect and determine the centroid object in $\mathrm{x}-\mathrm{y}$ coordinated.

\section{ACKNOWLEDGMENT}

The authors are grateful for the support by Universiti Teknikal Malaysia Melaka (UTeM) in conducting this research.

\section{REFERENCES}

Silvio M. Maeta, Samuel S. Bueno, Luiz G. Mirisola. “ Development of a VRML/Java Unmanned Airship Simulating Environment ".Journal.

Marcel Bergerman, Samuel Siqueira Bueno, Alberto Elfes "A Semi-Autonomous Robotic Airship for Environmental Monitoring Mission “ Journal.
Wikipedia, flight control http://en.wikipedia.org/wiki/flight_control_theory $\mathrm{Yu}$ Liu and Yueming $\mathrm{Hu}$ "Stability and Control Analysis Based on Airship Dynamic Modeling “ Journal.

EAI Specifications: http://www.vrml.org/

WorkingGroups/vrml-eai/Specification

Pospisilik Martin \& Adamek Milan. "Autonomous Airship as a Surrounding Monitor" Seminar ASR 2007 Instrument and Control (2007)

M Sulaiman, HNM Shah, MH Harun, WT Lim and MNFM Kazim. A 3D Gluing Defect Inspection System Using Shape-Based Matching Application from Two Cameras. International Review on Computers and Software (IRECOS ). 2013, 8(8), pp. 1997-2004.

Sulaiman, M., Shah, M. H. N., Harun, M. H., and Kazim, M. N. F. M. Defect Inspection System For Shape-Based Matching Using Two Cameras. Journal of Theoretical and Applied Information Technology (JATIT). 2014, 61(2), pp. 288-297.

MH Harun, M Sulaiman, HNM Shah, and WT Lim, "Shape - Based Matching: Defect Inspection of Glue Process in Vision System", Conference on Industrial Electronics and Applications (ISIEA), IEEE, pp. 53-57, 2011.

HNM Shah, M Sulaiman, AZ Shukor, MZA Rashid. Recognition of Butt Welding Joints using Background Subtraction Seam Path Approach for Welding Robot. International Journal of Mechanical \& Mechatronics Engineering. 2017, 17(01), pp. 57-62.

HNM Shah, M Sulaiman, AZ Shukor . Autonomous detection and identification of weld seam path shape position. 2017. pp 1-9.

MH Harun, M Sulaiman, HNM Shah, LW Teck. Shape-based matching: Defect inspection of glue process in vision system. IEEE Symposium on Industrial Electronics and Applications (ISIEA).2011. pp. 53-57.

WT Lim, M Sulaiman and HNM Shah, "Flexible Approach For Region Of Interest Creation For Shape-Based Matching In Vision System", Conference on Innovative Technologies in Intelligent Systems and Industrial Applications, IEEE, pp. 205-208, 2009.

WT Lim, M Sulaiman, HNM Shah. Shape-Based Matching: Defect Inspection of lue Process in Vision System. IEEE Symposium on Industrial Electronics and Applications. 2011.

HNM Shah, M Sulaiman, SNS Salim. Fuzzy Logic Approach for Mobile Robot in Intelligent Space. Journal-The Institution of Engineers. 2007.68.pp. 38-45

[16] HNM Shah, M Sulaiman, MZA Rashid, Z Kamis, NM Ali, MSM Aras, F Wasbari, Z Omar. A 3D Mapping of the Surrounding Object using StereoVision Technique. International Journal of Soft Computing. 2017, 12(1), pp. 59-65.

[17] HNM Shah, MZA Rashid, MF Abdollah, MN Kamarudin, Z Kamis, A Khamis. Detection of 
Mobile Object in Workspace Area. International Journal of Signal Processing, Image Processing and Pattern Recognition. 2016. 9(4). pp. 225-232.

[18] WT Lim, M Sulaiman, HNM Shah, R Omar, "Implementation of Shape-Based Matching Vision System in Flexible Manufacturing System", Journal of Engineering Science and Technology Review, Vol. 3, No 1, pp. 128- 135, 2011

[19] HNM Shah, M Sulaiman, AZ Shukor, MZA Rashid, MH Jamaluddin. "Review Paper on Vision Based Identification, Detection and Tracking of Weld Seams Path in Welding Robot Environment," Modern Applied Science, Vol. 10, No 2, pp. 83-89, 2016.

[20] HNM Shah, M Sulaiman, AZ Shukor, MZA Rashid. Recognition of Butt Welding Joints using Background Subtraction Seam Path Approach for Welding Robot. International Journal of Mechanical \& Mechatronics Engineering. 2017, 17(01), pp. 57-62.

[21] MH Harun, M Sulaiman and HNM Shah. Shape Based Matching: Application of Defect Detection. Malaysian Technical Universities International Conference on Engineering \& Technology (MUCEET). 2011.

[22] HNM Shah, MZA Rashid, YT Tam, "Develop and Implementation of Autonomous Vision Based Mobile Robot Following Human", International
Journal of Advanced Science and Technology, Vol. 51, No 2, pp. 81-91, 2013.

[23] HNM Shah, MZA Rashid, NMM Sobran. Autonomous Mobile Robot Vision Based System: Human Detection by Color. Journal of Theoretical and Applied Information Technology. 2013. PP. 17.

[24] HNM Shah, MZA Rashid, MF Abdollah, MN Kamarudin, Z Kamis, A Khamis. Detection of Mobile Object in Workspace Area. International Journal of Signal Processing, Image Processing and Pattern Recognition. 2016, 9(4), pp. 225-232.

[25] HNM Shah, MZA Rashid, Z Kamis, MN Kamarudin, MF Abdollah, A Khamis. Implementation of Object Recognition Based on Type of Vehicle Entering Main Gate. Indonesian Journal of Electrical Engineering and Computer Science. 2016, 3(2).

[26] MH Harun, M Sulaiman and HNM Shah. ShapeBased Matching: Application of Defect Detection. In: Proceedings of Malaysian Technical Universities International Conference on Engineering and Technology. 2011.

[27] MH Harun, M Sulaiman and HNM Shah. ShapeBased Matching: Application of Edge Detection Using Harris Point. International Conference on Robotic Automation System. 2011. pp. 1-5. 